\title{
RESEARCH
}

Open Access

\section{Biochemical and structural magnetic resonance imaging in chronic stroke and the relationship with upper extremity motor function}

Mohamed Mahmoud Mostafa', Eman Mahmoud Awad' ${ }^{1}$, Ahmed Mohamed Hazzou', Mohamed Khaled Ahmed Elewa', Tougan Taha Abdel Aziz and Dalia Maher Samy²*

\begin{abstract}
Background: Recovery of upper extremity (UE) motor function after stroke is variable from one to another due to heterogeneity of stroke pathology. Structural and biochemical magnetic resonance imaging of the primary motor cortex (M1) have been used to document reorganization of neural activity after stroke.

Objective: To assess cortical biochemical and structural causes of delayed recovery of UE motor function impairment in chronic subcortical ischemic stroke patients.
\end{abstract}

Methodology: A cross-sectional study with fifty patients were enrolled: thirty patients with chronic ( $>6$ months) subcortical ischemic stroke suffering from persistent UE motor function impairment (not improved group) and twenty patients with chronic subcortical ischemic stroke and improved UE motor function (improved group). We recruited a group of (16) age-matched healthy subjects. Single voxel proton magnetic resonance spectroscopy $(1 \mathrm{H}$ MRS) was performed to measure $\mathrm{N}$-acetylaspartate (NAA) and glutamate+glutamine (GIX) ratios relative to creatine $(\mathrm{Cr})$ in the precentral gyrus which represent M1 of hand area in both ipsilesional and contralesional hemispheres. Brain magnetic resonance imaging (MRI) to measure precentral gyral thickness is representing the M1of hand area. UE motor function assessment is using the Fugl Meyer Assessment (FMA-UE) Scale.

Results: The current study found that ipslesional cortical thickness was significantly lower than contralesional cortical thickness among all stroke patients. Our study found that ipsilesional NAA/Cr ratio was lower than contralesional NAA/Cr among stroke patients. UE and hand motor function by FMA-UE showed highly statistically significant correlation with ipsilesional cortical thickness and ipsilesional NAA/Cr ratio, more powerful with NAA/Cr ratio.

Conclusion: We concluded that persistent motor impairment in individuals with chronic subcortical stroke may be at least in part related to ipsilesional structural and biochemical changes in motor areas remote from infarction in form of decreased cortical thickness and NAA/Cr ratio which had the strongest relationship with that impairment.

Keywords: Stroke, Biochemical and structural, MRI, Upper extremity motor function

\footnotetext{
* Correspondence: dalia84maher@yahoo.com

${ }^{2}$ El Sahel Teaching Hospital, Cairo 11697, Egypt

Full list of author information is available at the end of the article
}

\section{Springer Open}

( ) The Author(s). 2020 Open Access This article is licensed under a Creative Commons Attribution 4.0 International License, which permits use, sharing, adaptation, distribution and reproduction in any medium or format, as long as you give appropriate credit to the original author(s) and the source, provide a link to the Creative Commons licence, and indicate if changes were made. The images or other third party material in this article are included in the article's Creative Commons licence, unless indicated otherwise in a credit line to the material. If material is not included in the article's Creative Commons licence and your intended use is not permitted by statutory regulation or exceeds the permitted use, you will need to obtain permission directly from the copyright holder. To view a copy of this licence, visit http://creativecommons.org/licenses/by/4.0/. 


\section{Introduction}

Motor impairment of one side of the body is a major cause of disability in activities of daily living. Recovery from strokes varies from one patient to another due to the heterogeneity of the stroke pathology and rehabilitation strategies. While most stroke patients recover spontaneously, many are left with permanent neurological impairments [1].

Understanding the brain pathologies associated with upper extremity (UE) impairment after stroke, the underlying mechanisms of injury, and the processes associated with recovery is important for achieving good recovery and successful rehabilitation. Advancements in neuroimaging technology have made this possible. Structural and biochemical brain imaging of primary motor cortices has been used to document the reorganization of neural activity after stroke. Ipsilesional and contralesional primary motor cortices, as well as the dorsal premotor cortex, have been identified as areas that can undergo substantial post-stroke neuroplasticity [2].

Single voxel proton magnetic resonance spectroscopy $(1 \mathrm{H}-\mathrm{MRS})$ is a non-invasive tool to measure the levels of certain metabolites. In acute stroke, the presence of a measurable lactate peak demonstrates a shift from aerobic to anaerobic metabolism in the brain. Previous studies have shown that this is a temporary effect, and levels return to an almost undetectable state within 3 weeks [3].

Biochemical changes have also been observed chronically within ipsilesional normal-appearing grey matter and have been associated with morphological changes in stroke patients [4].

$\mathrm{N}$-Acetylaspartate (NAA) is a metabolite found exclusively in neurons and their processes. It is considered a putative marker of their integrity with specific roles in the central nervous system, including myelin synthesis, neuronal energetics, neuronal osmoregulation, and axonal-glial signaling [5].

In individuals with chronic stroke, lower NAA and higher myo-inositol (an astrocyte marker) concentrations have been reported within ipsilesional and contralesional primary motor cortices [6].

A positive correlation has been drawn between NAA, glutamate (Glu: the principle excitatory neurotransmitter in the human central nervous system), and UE function in a chronic stroke population [7].

Structural changes in regional cortical thickness have also been observed in individuals in the subacute phase (3 months of recovery) after subcortical ischemic stroke and have been linked to functional activation changes in individuals with chronic stroke $[8,9]$.

The purpose of this study is to assess cortical, biochemical, and structural causes of delayed recovery of UE motor function impairment in patients with chronic, subcortical ischemic stroke.

\section{Subjects and methods}

A cross-sectional study with fifty patients were enrolled: thirty patients with chronic (> 6 months) subcortical ischemic stroke suffering from persistent upper extremity motor function impairment (not improved group) and twenty patients with chronic subcortical ischemic stroke and improved upper extremity motor function (improved group).

All patients were recruited from El Sahel Teaching Hospital outpatient clinics.

\section{Inclusion criteria}

1. Eligible right handed patients aged between 50 and 70 years old.

2. Single clinically diagnosed chronic (> 6 months) subcortical ischemic stroke presented with UE motor impairment at the acute stage.

3. Radiologically apparent healthy precentral gyrus grey matter.

\section{Exclusion criteria}

1. Patients with disturbed consciousness level.

2. Patients with aphasia, cognitive impairment, UE apraxia, sensory deficit, or ataxia.

3. Patients with history of previous stroke.

4. Patients who had any contraindication for MRI or $1 \mathrm{H}-\mathrm{MRS}$.

5. Patients who underwent neuro-rehabilitation through transmagnetic brain stimulation or transcranial direct current brain stimulation.

6. Patients with uncontrolled diabetes mellitus.

7. Patients with chronic renal or hepatic failure.

We recruited right-handed age-matched (16) healthy subject group to be references for our results regarding Egyptian people.

\section{Methods}

All participants subjected to:

1. Full medical history and neurological examination.

2. Routine lab and imaging.

3. (1H-MRS) to measure NAA and Glx concentration as ratios of their peak heights to $\mathrm{Cr}$ peak height as a stable internal reference (NAA/Cr and $\mathrm{Glx} / \mathrm{Cr}$ ) in ipsilesional and contralesional precentral gyrus which represent the M1 of hand area (landmark of precentral gyrus), determined anatomically by the area that faces and forms the "middle knee" of the central sulcus, located just at the cross point between the precentral sulcus and the central sulcus, which is topographically located at the level of the distal end 
of the superior frontal sulcus and is therefore also visible on the cortical surface [10] (Fig. 1).

4. MRI brain to (a) quantify cortical thickness of the precentral gyrus representing the M1of hand area in both ipsilesional and contralesional hemispheres through Picture Archiving and Communication System (PACS), presented in millimeters ( $\mathrm{mm}$ ) and (b) assess white matter disease by fazekas scale which divides the white matter in periventricular (PVWM) and deep white matter (DWM) through fluid attenuated recovery (FLAIR) film with the higher score means more severity.

5. UE motor function assessment using the Fugl Meyer Assessment (FMA-UE) Scale [11].

6. Modified rankin scale (mRS) to assess disability degree in stroke patients ranging from 0 to 5 with higher score indicating more disability [12].

\section{Procedures}

1. MRI scans were completed on Ingenia 1.5 Tesla Philips, and a high-resolution axial T1-weighted (TR/TE 256/2 ms, flip angle $80^{\circ}$ ), axial flair (TR/TE $11000 / 140$ ms, flip angle $90^{\circ}$ ), sagittal T1 (TR/TE $166 / 2 \mathrm{~ms}$, flip angle $80^{\circ}$ ), and coronal T2 scan (TR/TE 6600/110 ms, flip angle $90^{\circ}$ ) were performed on each enrolled participant. In addition, single voxel 1H-MRS (TR/TE = 2000/31, 2000/144 ms) was performed to measure metabolite ratios. A single $15-\mathrm{mm} \times 15 \mathrm{~mm} \times 15 \mathrm{~mm}$ voxel was centered over the M1 of hand area in both ipsilesional and contralesional hemispheres. Localization of hand representation for each individual and corresponding voxel placement was determined using known anatomical coordinates described above.

2. Assessment of affected UE motor function by FMA-UE with score ranges from 0 to 66 from which 14 points represent hand motor function with higher scores indicating better motor function.

In our study, the improved group had score from 64 to 66 for UE motor function and 14 for hand, the not improved group with score $<64$ for UE and $<14$ for the hand.

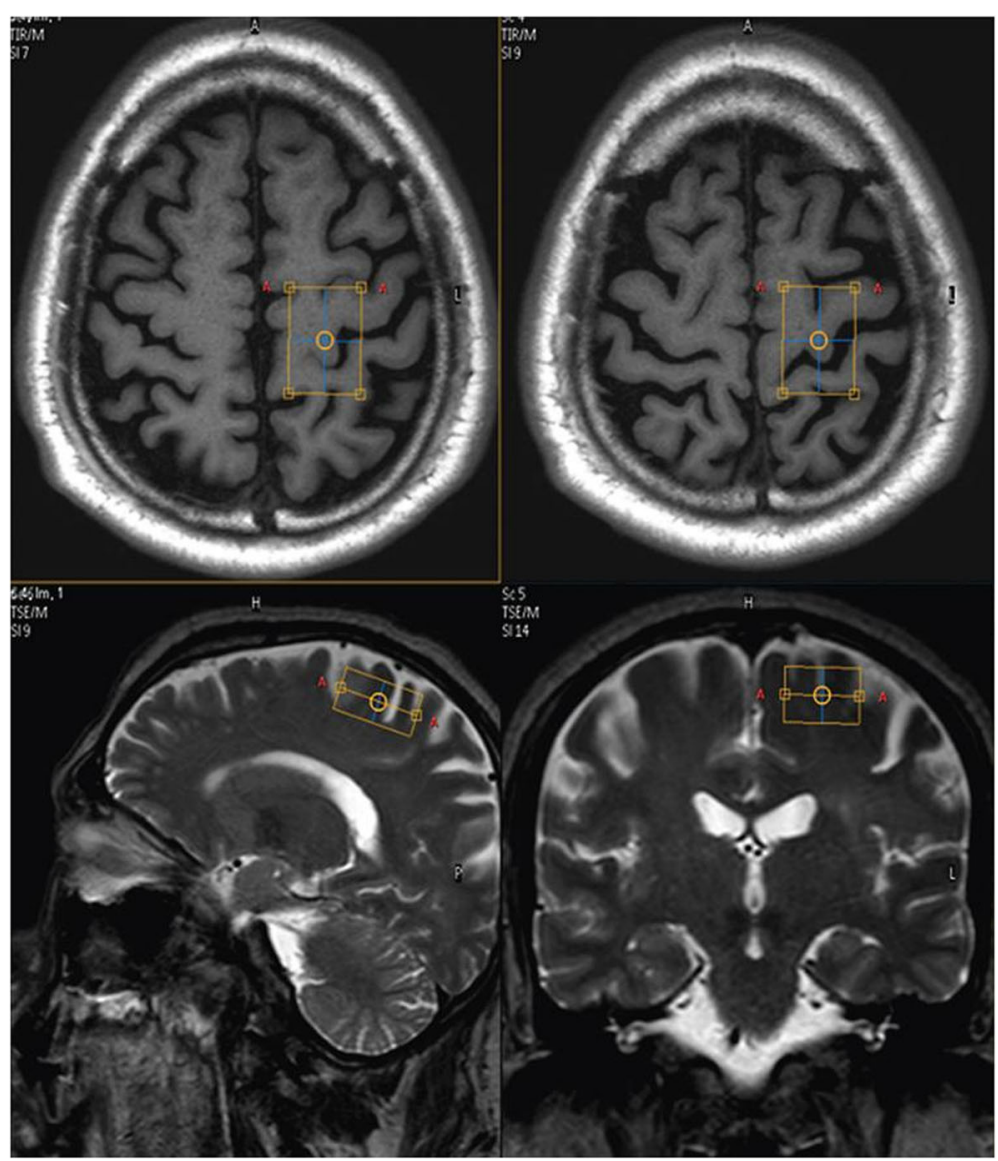

Fig. 1 Precentral gyrus, which represents the M1 of hand area (landmark of precentral gyrus), determined anatomically by the area that faces and forms the "middle knee" of the central sulcus, located just at the cross point between the precentral sulcus and the central sulcus, which is topographically located at the level of the distal end of the superior frontal sulcus and is therefore also visible on the cortical surface 


\section{Statistical analysis}

The collected data was revised, coded, tabulated, and introduced to a PC using Statistical package for Social Science (SPSS 20). Data was presented, and suitable analysis was done according to the type of data obtained for each parameter.

i. Descriptive statistics

1. Mean, standard deviation ( \pm SD), and range for parametric numerical data, while median and interquartile range (IQR) for non-parametric numerical data.

2. Frequency and percentage of non-numerical data.

ii. Analytical statistics:

1. Paired $t$ test

2. Student $T$

3. Chi-square test

4. Correlation analysis (using Pearson's and Spearman's method)

5. Regression coefficient for determination of the strongest predictor for FMA- UE.

" $P$ value: level of significance

$-P>0.05$ : non-significant (NS).

$-P<0.05$ : significant $(\mathrm{S})$.

$-P<0.01$ : highly significant (HS).

\section{Results}

\section{Descriptive data}

Our study groups included 24 males (48\%) and 26 females $(52 \%)$. The age of the patients ranged from 50 to 70 years with a mean age of $59.04 \pm 6.104$.

All patients were assessed with brain MRI. Twenty six cases (52\%) had left subcortical infarctionm and 24 cases (48\%) had right subcortical infarction. Among them, 15 cases (30\%) had presented with capsular infarction, 11 cases (22\%) had presented with pontine infarction, 5 cases $(10 \%)$ had presented with thalamic infarctions, two cases $(4 \%)$ had a basal ganglia infarction, and 17 cases (34\%) had other subcortical area infarctions.

\section{Comparisons}

Comparing the demographic data and risk factors between the two study groups, neither age, genderm nor risk factors had statistically significant difference between both groups.

Both groups also were matched as regard the radiological data, but significant difference was found in fazekas (PVWM) score regarding score zero among the two groups.
As regards to the improved group, there was no statistically significant difference between ipsilesional and contralesional (cortical thickness, NAA/Cr ratio, and $\mathrm{Glx} / \mathrm{Cr}$ ratio), while in not improved group, there was a highly statistically significant difference between ipsilesional and contralesional (cortical thickness, NAA/Cr ratio, and $\mathrm{Glx} / \mathrm{Cr}$ ratio) with $P=(0.002,<0.001$, and $<$ 0.001 ), respectively.

As regards to all stroke patients, there was a statistically significant difference between ipsilesional and contralesional cortical thickness, and a highly statistically significant difference between ipsilesional and contralesional (NAA/Cr ratio and $\mathrm{Glx} / \mathrm{Cr}$ ratio) with $P=(0.011,0.001$, and 0.009), respectively. All details are in Table 1.

As regards ipsilesional cortical thickness, there was a highly statistically significant difference $P=0.007$ between the not improved and the improved groups with mean $2.48 \mathrm{~mm}$ in the not improved group vs $2.87 \mathrm{~mm}$ in the improved group. As regards to NAA/Cr ratio, there was a highly statistically significant difference $P=0.001$ between the not improved and the improved groups with mean 1.54 in the not improved group vs 1.83 in the improved group, but GLX/Cr ratio had no statistically significant difference between the two groups. All details are in Table 2.

There was no statistically significant difference between both groups neither in contralesional cortical thickness, NAA/Cr ratio, nor GLX /Cr ratio although the mean of the not improved group higher than that of the improved group as regards to $\mathrm{NAA} / \mathrm{Cr}$ ratio and GLX/Cr ratio.

Table 1 Comparison between ipsilesional and contralesional cortical thickness and metabolite ratios in the improved, not improved, and all stroke groups

\begin{tabular}{|c|c|c|c|c|c|c|}
\hline \multirow[t]{2}{*}{ Improved $\boldsymbol{N}=20$} & \multicolumn{2}{|c|}{ Ipsilesional } & \multicolumn{2}{|c|}{ Contralesional } & \multicolumn{2}{|c|}{ Paired $\boldsymbol{t}$ test } \\
\hline & Mean & SD & Mean & SD & $\boldsymbol{p}$ value & sig. \\
\hline Cortical thickness & 2.87 & 0.45 & 2.89 & 0.48 & 0.854 & NS \\
\hline NAA/Cr ratio & 1.83 & 0.28 & 1.86 & 0.37 & 0.750 & NS \\
\hline GLX/Cr ratio & 0.25 & 0.15 & 0.25 & 0.16 & 0.980 & NS \\
\hline \multicolumn{7}{|l|}{ Not improved, $N=30$} \\
\hline Cortical thickness & 2.48 & 0.50 & 2.77 & 0.46 & 0.002 & $\mathrm{HS}$ \\
\hline NAA/Cr ratio & 1.54 & 0.29 & 1.86 & 0.24 & 0.000 & $\mathrm{HS}$ \\
\hline $\mathrm{GLX} / \mathrm{Cr}$ ratio & 0.21 & 0.17 & 0.34 & 0.20 & 0.000 & $\mathrm{HS}$ \\
\hline \multicolumn{7}{|c|}{ All stroke group, $N=50$} \\
\hline Cortical thickness & 2.63 & 0.50 & 2.81 & 0.47 & 0.011 & S \\
\hline NAA/Cr ratio & 1.65 & 0.31 & 1.86 & 0.30 & 0.001 & $\mathrm{HS}$ \\
\hline GLX/Cr ratio & 0.23 & 0.16 & 0.31 & 0.19 & 0.009 & HS \\
\hline
\end{tabular}

Paired $t$ test, $S$ significant, $H S$ highly significant, $N S$ non-significant, $N A A / C r \mathrm{~N}^{-}$ acetylaspartate/creatine ratio, $G L X / C r$ glutamate/creatine ratio 
Table 2 Comparison in ipsilesional (cortical thickness and metabolite ratios) between the two study groups

\begin{tabular}{|c|c|c|c|c|c|c|}
\hline & \multicolumn{2}{|c|}{ Not improved } & \multicolumn{2}{|c|}{ Improved } & \multicolumn{2}{|l|}{$\boldsymbol{t}$ test } \\
\hline & Mean & SD & Mean & SD & $\boldsymbol{p}$ value & sig. \\
\hline Ipsilesional cortical thickness & 2.48 & 0.49 & 2.87 & 0.44 & 0.007 & HS \\
\hline Ipsilesional NAA/ Cr ratio (height) & 1.54 & 0.29 & 1.83 & 0.28 & 0.001 & HS \\
\hline Ipsilesional GLX/Cr ratio (height) & 0.21 & 0.17 & 0.25 & 0.15 & 0.346 & NS \\
\hline
\end{tabular}

t test, $H S$ highly significant, $N S$ non-significant, $N A A / C r$ N-acetylaspartate/creatine ratio, $G L X / C r$ glutamate/creatine ratio

\section{Correlations}

As regards to ipslesional cortical thickness, there was highly significant positive correlation with FMA-UE and FMA of the hand with $P=0.002$ and 0.006 , respectively and highly significant negative correlation with $\mathrm{mRS}$ with $P=0.001$; also, ipsilesional NAA/Cr ratio had a highly significant positive correlation with FMA-UE and FMA of the hand with $P=0.006$ and 0.008 , respectively and highly significant negative correlation with $\mathrm{mRS}$ with $P=0.001$, but contralesional cortical thickness, contralesional $\mathrm{NAA} / \mathrm{Cr}$ ratio, and both ipslesional and contralesional $\mathrm{GLX} / \mathrm{Cr}$ ratios had no any correlations with mRS, FMA-UE, and FMA of the hand. All details are in Table 3.

As regards to regression coefficient test for FMA -UE there was highly statistically significance with ipsilesional cortical thickness and ipsilesional $\mathrm{NAA} / \mathrm{Cr}$ ratio, more powerful with $\mathrm{NAA} / \mathrm{Cr}$ ratio which means that every one point change in $\mathrm{NAA} / \mathrm{Cr}$ ratio associated with 27.58 points increase in FMA score. Also regarding regression coefficient test for FMA of the hand there was highly statistically significance with ipsilesional cortical thickness and significant correlation with ipsilesional NAA/ $\mathrm{Cr}$ ratio, more powerful with $\mathrm{NAA} / \mathrm{Cr}$ ratio which means that every one point change in NAA/Cr ratio associated with 5.76 points increase in FMA score. Table 4 shows details.

As regards to time from onset, there was highly significant positive correlation with ipsilesional NAA/Cr ratio with $P=0.003$, and no any correlation with ipsilesional, contralesional cortical thickness, contralesional NAA/Cr ratio, ipsilesional, contralesional $\mathrm{GLX} / \mathrm{Cr}$ ratio, $\mathrm{mRS}$, FMA-UE, and FMA of the hand.

As regards to fazekas score (PVWM and DWM), there was no significant correlation with mRS.

\section{Data of healthy subjects}

We recruited 16 healthy subjects to be references for our results regarding Egyptian people with age range from 50-66 years and mean of age was $57.25 \pm 4.99$ SD years, mean of right cortical thickness was $2.96 \pm 0.31$ $\mathrm{SD} \mathrm{mm}$, and that of left cortical thickness was $2.96 \pm$ $0.4 \mathrm{SD} \mathrm{mm}$. As regards to NAA/Cr ratio, the mean of right hemisphere was $2.01 \pm 0.34 \mathrm{SD}$ while that of left hemisphere was $1.89 \pm 0.32 \mathrm{SD}$. As regards to $\mathrm{GLX} / \mathrm{Cr}$ ratio, the mean of right hemisphere was $0.3 \pm 0.19 \mathrm{SD}$ while that of the left hemisphere was $0.31 \pm 0.16 \mathrm{SD}$ with no significant differences between the two hemispheres in healthy subjects for any of the variables.

\section{Discussion}

In the current study, we assessed the $\mathrm{NAA} / \mathrm{Cr}$ and $\mathrm{Glx} / \mathrm{Cr}$ ratios in the M1 of the hand area in individuals in the chronic stage of stroke recovery. These metabolites were selected for two reasons: first, changes in both of these metabolites have been reported in individuals with chronic stroke and were also associated with UE impairment. Second, an observed decrease in cortical thickness is most likely explained by neuronal death, which can be assessed using NAA as a marker of neuronal integrity $[4,6,13]$.

As regards UE motor function, we found a high significant correlation between the ipsilesional $\mathrm{NAA} / \mathrm{Cr}$

Table 3 Correlation of cortical thickness and metabolites ratios with mRS, FMA-UE, and FMA of the hand

\begin{tabular}{|c|c|c|c|c|c|c|c|}
\hline \multicolumn{2}{|c|}{$\begin{array}{l}\text { Whole sample, } \boldsymbol{N}= \\
50\end{array}$} & \multirow{2}{*}{$\begin{array}{l}\text { Ipsilesion-al } \\
\text { cortical thickness } \\
0.429\end{array}$} & \multirow{2}{*}{$\begin{array}{l}\text { Contrale-sional } \\
\text { cortical thickness }\end{array}$} & \multirow{2}{*}{$\begin{array}{l}\text { Ipsilesional NAA/Cr } \\
\text { ratio (height) } \\
0.383\end{array}$} & \multirow{2}{*}{$\begin{array}{l}\text { Contrale-sional NAA/ } \\
\text { Cr ratio (height) } \\
0.026\end{array}$} & \multirow{2}{*}{$\begin{array}{l}\text { Ipsilesional GLX/Cr } \\
\text { ratio (height) } \\
0.148\end{array}$} & \multirow{2}{*}{$\begin{array}{l}\text { Contrale-sional GLX/ } \\
\text { Cr ratio (height) } \\
-0.154\end{array}$} \\
\hline FMA-UE > & Rs & & & & & & \\
\hline 6 months & $p$ value & 0.002 & 0.507 & 0.006 & 0.856 & 0.306 & 0.285 \\
\hline \multirow{2}{*}{$\begin{array}{l}\text { FM hand > } \\
6 \text { months }\end{array}$} & Rs & 0.384 & 0.074 & 0.369 & 0.060 & 0.134 & -0.154 \\
\hline & $p$ value & 0.006 & 0.609 & 0.008 & 0.677 & 0.354 & 0.284 \\
\hline \multirow[t]{2}{*}{$\mathrm{mRS}$} & Rs & -0.468 & -0.022 & -0.456 & -0.106 & -0.217 & 0.180 \\
\hline & $p$ value & 0.001 & 0.878 & 0.001 & 0.465 & 0.130 & 0.212 \\
\hline
\end{tabular}


Table 4 Regression coefficient test for FMA-UE and FMA of the hand

\begin{tabular}{|c|c|c|c|c|}
\hline FMA-UE > 6 months & Regression coefficient & $95 \% \mathrm{Cl}$ & $\boldsymbol{p}$ value & sig. \\
\hline Ipsilesional cortical thickness & 19.20 & $6.84-31.55$ & 0.003 & HS \\
\hline Ipslesional NAA/Cr ratio & 27.58 & $7.59-47.57$ & 0.008 & HS \\
\hline \multicolumn{5}{|l|}{ FMA hand $>6$ months } \\
\hline Ipsilesional cortical thickness & 5.10 & $2.19-8.01$ & 0.001 & HS \\
\hline Ipsilesional NAA/Cr ratio & 5.76 & $1.05-10.47$ & 0.018 & $S$ \\
\hline
\end{tabular}

$S$ significant, $H S$ highly significant, $N A A / C r$ N-acetylaspartate/creatine ratio, GLX/Cr glutamate/creatine ratio, FMA-UE Fugl Meyer Assessment-upper extremity, FMA Fugl Meyer Assessment

ratio and both the FMA-UE score and FMA score of the hand. This is in agreement with Jones et al. and Craciunas et al. [3, 7].

Regarding the contralesional NAA/Cr ratio, our results showed no correlation with either the FMA-UE score or FMA score of the hand. These results are contrary to the findings of Goldzik et al. [14], who found a positive correlation between a contralesional prefrontal NAA/Cr ratio and recovery after stroke. However, the researchers did not relate the area where the metabolite ratios were measured to a specific clinical deficit in the patients studied.

While the ipsilesional $\mathrm{Glx} / \mathrm{Cr}$ ratio showed a highly significant reduction in comparison with the contralesional $\mathrm{Glx} / \mathrm{Cr}$ ratio in the stroke patients, we observed no association between this decrease and UE motor function, which is consistent with Jones et al.'s study [3] but contrary to Cirstea et al.'s study [6]. The latter found that the Glx concentration in motor areas was correlated with arm motor impairment in individuals with chronic stroke; this disagreement may be due to the small sample size used by the previous study compared to the sample size in the current study.

Other studies support the hypothesis that a decrease in ipsilesional Glx levels is associated with UE motor performance based on reported changes in intracortical excitability after a stroke, as well as a correlation found between the Glx concentration in the motor cortex and global motor cortical excitability (assessed by transcranial magnetic stimulation), which predict motor recovery after stroke [15-18]. However, further investigations are needed to resolve this issue.

In our study, we found a high significant correlation between ipsilesional cortical thickness and the FMAUE score and FMA score of the hand. This finding is in agreement with Jones et al. [3] and Gauthier et al. [19], who noted that decreases in grey matter density in non-infarcted motor regions in individuals with chronic stroke correlates with the WMFT of UE motor function.

With regard to the regression coefficient test for the FMA-UE, it was highly statistically significant with both ipsilesional cortical thickness and the ipsilesional NAA/ $\mathrm{Cr}$ ratio, but it was more powerful with the latter; every 1-point change in the NAA/Cr ratio was associated with a 27.58-point increase in the FMA-UE score.

In addition, the regression coefficient test for the FMA of the hand showed high statistical significance with both ipsilesional cortical thickness and the ipsilesional $\mathrm{NAA} / \mathrm{Cr}$ ratio, but it was more powerful with $\mathrm{NAA} / \mathrm{Cr}$ ratio, as every 1-point change in this ratio was associated with an increase of 5.76 points in the FMA score of the hand. This finding is in agreement with Jones et al. [3], who concluded that the NAA concentration, a marker for neuronal integrity, in spared ipsilesional motor cortical areas that may contribute to UE motor function in individuals in the chronic stage post-stroke. So, while stroke impairs both the structure and biochemistry in the ipsilesional hemisphere, our data suggest that ipsilesional cortical NAA in the motor hand area has the strongest relationship with motor function of the UE and the hand, which may have an impact on future pharmacotherapies and rehabilitation methods in treating stroke.

Our study posits a highly significant reduction in the ipsilesional $\mathrm{NAA} / \mathrm{Cr}$ ratio compared to the contralesional NAA/Cr ratio among stroke patients and in the group that showed no improvement. However, no significant difference was found among the group that showed improvement or in the hemispheres of healthy subjects.

We also observed a decrease in the ipsilesional and contralesional NAA/Cr ratios in all stroke patients (with mean values of 1.65 and 1.86, respectively) in comparison with healthy subjects (with mean values of 2.01 in the right hemisphere and 1.89 in the left hemisphere) for healthy subjects. Our results are consistent with previous findings in stroke studies $[3,6,7]$.

Because these biochemical changes were observed in brain regions remote from the infarct, it is possible that diaschisis could explain these results. Diaschisis is a common dysfunction after stroke caused by the impairment of function in intact brain regions connected to, but distant from the area of infarct [20]. 
The current study also revealed decrease in ipsilesional $\mathrm{Glx} / \mathrm{Cr}$ ratio among stroke patients in comparison with healthy subjects with a mean of 0.23 versus 0.30 and 0.31 in the right and left healthy hemispheres, respectively.

Our study found highly significant reduction in ipsilesional $\mathrm{Glx} / \mathrm{Cr}$ ratio than the contralesional side among stroke patients and also among not improved group, but no significant difference between ipsilesional $\mathrm{Glx} / \mathrm{Cr}$ ratio and contralesional $\mathrm{Glx} / \mathrm{Cr}$ ratio among improved group or among both hemispheres in healthy subjects, which is consistent with Jones et al. [3].

A possible mechanism that may explain our findings of Glx and NAA concentration is neuronal death. Neuronal death following ischemic stroke is often attributed to improper homeostatic balance of Glu, as Glu cannot be taken up by either neurons or astrocytes, and levels of Glu rapidly rise in the extracellular space results in a cascade of cellular events such as intracellular calcium increase and mitochondrial dysfunction that ultimately leads to cellular death [21,22] which in turn explains the metabolite ratios decrease in our study.

The current study also found that ipslesional cortical thickness was significantly lower than contralesional cortical thickness among all stroke patients, and this significant difference was high among not improved group, but no significant difference among improved group or our healthy subjects group which is in agreement with Jones et al. [3] and Zhang et al. [23] who demonstrated that although the exact mechanisms for structural impairment of the ipsilesional M1 in subcortical stroke remain unclear, it is possible that retrograde degeneration may account for this finding.

For example, retrograde trans-synaptic degeneration in the retinal nerve fiber layer has been observed in patients with occipital lobe infarction [24].

Our results are also consistent with Duering et al. [25] who found that the integrity of the corticospinal tract potentially associated with the extent of focal cortical thinning in patients with incident subcortical infarcts.

Our study also observed that the mean of ipsilesional cortical thickness among not improved group was the lowest in comparison with improved group and right and left hemispheres of normal healthy group $(2.48 \mathrm{~mm}$, $2.87 \mathrm{~mm}, 2.96$, and $2.97 \mathrm{~mm}$ ), respectively, which consistent with Zhang et al. [23] who found that M1 cortical thinning is present even in stroke patients with improved global motor function, suggesting that motor recovery in these patients may be mainly associated with functional reorganization.

The limited use of the paretic limb in the not improved group may also explain our results about the thinning of the ipsilesional cortical thickness.

The current study revealed no statistically significant differences between improved and not improved groups as regards to age, sex, risk factors, and radiological data except for fazekas scale (PVWM) which had statistically significant difference between the two study groups, After sub grouping by post hoc test, this difference was related to PVWM score 0 only as 4 of our improved patients had score 0 , and no patients of the not improved group had this score, with no differences regarding other PVWM scores between the two groups.

As regards to time from onset, there was highly significant positive correlation with ipsilesional NAA/Cr ratio, and this is consistent with several reports which documented NAA concentration recovery in gray matter after stroke, suggesting that some degree of reversible neuronal injury accompanies ischemia [26].

In the current study, no correlation found between time from onset and contralesional $\mathrm{NAA} / \mathrm{Cr}$ ratio which is inconsistent with Cristea et al. [6]who found positive correlation between the two items; as in that study, the range of duration post-stroke was wide (11-106 months) versus (7-18 months) in our study.

Time from onset also had no correlation with ipsilesional cortical thickness which in agreement with Brodtmann et al. [8] who found no change in ipsilesional cortical thickness overtime post-stroke.

In our study, no correlation noticed between time after stroke with mRS, FMA-UE score, and FMA score of the hand, and that is because recovery after stroke depends upon other multiple factors such as baseline function and disability, early mobilization, and if patients underwent physiotherapy or not.

Neither contralesional cortical thickness nor ipsilesional and contralesional GLX/Cr ratio had any correlation with time from onset that is may attributed to our narrow range regarding time after stroke.

As regards to fazekes scale, there was no any correlations with disability after stroke which disagree with Huo et al. [27] and Held et al. [28]; as in the current study, only $10 \%$ of patients had the higher fazekas score regarding PVWM and 0\% regarding DWM which in agreement with Zerna et al. [29] who concluded that higher fazekas scale scores correlated with post-stroke outcome.

\section{Conclusion}

Our study concluded that persistent motor impairment in individuals with chronic subcortical stroke may be at least in part related to ipsilesional structural and biochemical changes in motor areas remote from infarction in form of decreased cortical thickness and NAA/Cr ratio which had the strongest relationship with that impairment.

\section{Recommendations}

1. Availability of software to measure absolute concentrations of brain metabolites. 
2. Use of higher resolution MRI devices (more than $1.5 \mathrm{~T}$ ) in future studies for more detailed structural and biochemical data.

3. Start study from acute phase and follow-up in the chronic phase.

4. Larger studies determine how rehabilitation methods may be related to metabolites or cortical thickness in chronic stroke.

\section{Abbreviations}

1H-MRS: Proton magnetic resonance spectroscopy; Cho: Choline; CNS: Central nervous system; Cr: Creatine; CSF: Cerebrospinal fluid; DWM: Deepwhite matter; FLAIR: Fluid-Attenuated Inversion Recovery; FMA: Fugl Meyer Assessment; FMA-UE: Fugl Meyer Assessment-upper extremity; fMRl: Functional magnetic resonance imaging; GLU: Glutamate; Glx: Glutamate and glutamine; IQR: Interquartile range; Lip: Lipids; M1: Primary motor cortex; ml: Myo-inositol; Mm: Millimeters; MRA: Magnetic resonance angiography; mRS: Modified Rankin Scale; NAA: N-Acetylaspartate; PWWM: Periventricular white matter; SD: Standard deviation; TE: Echo time; tNAA: Total N-acetylaspartate; TR: Repitition time; UE: Upper extremity

\section{Acknowledgements}

Not applicable.

\section{Authors' contributions}

All authors read and approved the final manuscript.

\section{Funding}

No fund.

\section{Availability of data and materials}

Not applicable

\section{Ethics approval and consent to participate}

An approval of the study was obtained from Ain Shams University academic and ethical committee.

All participants signed an informed consent for participation in the study.

\section{Consent for publication}

Not applicable

\section{Competing interests}

The authors declare that they have no competing interests.

\section{Author details}

${ }^{1}$ Faculty of Medicine, Ain Shams University, Cairo, Egypt. ${ }^{2}$ El Sahel Teaching Hospital, Cairo 11697, Egypt.

Received: 11 February 2020 Accepted: 14 May 2020

Published online: 03 June 2020

\section{References}

1. Chang WH, Park CH, Kim DY, Shin YI, Ko MH, Lee A, et al. Cerebrolysin combined with rehabilitation promotes motor recovery in patients with severe motor impairment after stroke. BMC neurology. 2016 Dec;16(1):31.

2. Dimyan MA, Cohen LG. Neuroplasticity in the context of motor rehabilitation after stroke. Nature Reviews Neurology. 2011 Feb:7(2):76.

3. Jones PW, Borich MR, Vavsour I, Mackay A, Boyd LA. Cortical thickness and metabolite concentration in chronic stroke and the relationship with motor function. Restorative neurology and neuroscience. 2016 Jan 1;34(5):733-46.

4. Maniega SM, Cvoro V, Chappell FM, Armitage PA, Marshall I, Bastin ME, et al. Changes in NAA and lactate following ischemic stroke: a serial MR spectroscopic imaging study. Neurology. 2008;71(24):1993-9.

5. Ahmed AB, Cirstea CM. Positive effect of impairment-oriented training on $\mathrm{N}$-acetylaspartate levels of ipsilesional motor cortex in subcortical stroke: a case study. Int J Phys Med Rehabil. 2016:4(325):2.

6. Cirstea CM, Brooks WM, Craciunas SC, Popescu EA, Choi IY, Lee P, et al. Primary motor cortex in stroke: a functional MRI-guided proton MR spectroscopic study. Stroke. 2011 Apr;42(4):1004-9.
7. Craciunas SC, Brooks WM, Nudo RJ, Popescu EA, Choi IY, Lee P, et al. Motor and premotor cortices in subcortical stroke: proton magnetic resonance spectroscopy measures and arm motor impairment. Neurorehabil Neural Repair. 2013;27(5):411-20.

8. Brodtmann A, Pardoe H, Li Q, Lichter R, Ostergaard L, Cumming T. Changes in regional brain volume three months after stroke. Journal of the neurological sciences. 2012 Nov 15;322(1-2):122-8.

9. Schaechter JD, Moore Cl, Connell BD, Rosen BR, Dijkhuizen RM. Structural and functional plasticity in the somatosensory cortex of chronic stroke patients. Brain. 2006;129(10):2722-33.

10. Ribas G. The cerebral sulci and gyriReview article. Neurosurg Focus. 2010;28(2):E2.

11. Fugl-Meyer AR, Jääskö L, Leyman I, Olsson S, Steglind S. The post-stroke hemiplegic patient. 1. a method for evaluation of physical performance. Scandinavian journal of rehabilitation medicine. 1975;7(1):13-31.

12. Wilson JL, Hareendran A, Hendry A, Potter J, Bone I, Muir KW. Reliability of the modified Rankin Scale across multiple raters: benefits of a structured interview. Stroke. 2005;36(4):777-81.

13. Cirstea CM, Savage CR, Nudo RJ, Cohen LG, Yeh HW, Choi IY, et al. Handgrip-related activation in the primary motor cortex relates to underlying neuronal metabolism after stroke. Neurorehabilitation and neural repair. 2014 Jun;28(5):433-42

14. Glodzik-Sobanska L, Li J, Mosconi L, Slowik A, Walecki J, Szczudlik A, et al. Prefrontal $\mathrm{N}$-acetylaspartate and poststroke recovery: a longitudinal proton spectroscopy study. Am J Neuroradiol. 2007;28(3):470-4.

15. Tarkka IM, Könönen M, Pitkänen K, Sivenius J, Mervaala E. Alterations in cortical excitability in chronic stroke after constraint-induced movement therapy. Neurol Res. 2008;30(5):504-10.

16. Stagg CJ, Bestmann S, Constantinescu AO, Moreno Moreno L, Allman C, Mekle $\mathrm{R}$, et al. Relationship between physiological measures of excitability and levels of glutamate and GABA in the human motor cortex. The Journal of physiology. 2011 Dec;589(23):5845-55.

17. Carmichael ST. Brain excitability in stroke: the yin and yang of stroke progression. Archives of neurology. 2012 Feb 13;69(2):161-7.

18. Jung SH, Kim YK, Kim SE, Paik NJ. Prediction of motor function recovery after subcortical stroke: case series of activation PET and TMS studies. Annals of rehabilitation medicine. 2012 Aug;36(4):501.

19. Gauthier LV, Taub E, Mark WW, Barghi A, Uswatte G. Atrophy of spared gray matter tissue predicts poorer motor recovery and rehabilitation response in chronic stroke. Stroke. 2012 Feb;43(2):453-7.

20. Dancause N, Barbay S, Frost SB, Mahnken JD, Nudo RJ. Interhemispheric connections of the ventral premotor cortex in a new world primate. J Comp Neurol. 2007;505(6):701-15.

21. Hazell AS. Excitotoxic mechanisms in stroke: an update of concepts and treatment strategies. Neurochem Int. 2007;50(7-8):941-53.

22. Puig B, Brenna S, Magnus T. Molecular communication of a dying neuron in stroke. International journal of molecular sciences. 2018 Sep;19(9):2834.

23. Zhang J, Meng L, Qin W, Liu N, Shi FD, Yu C. Structural damage and functional reorganization in ipsilesional $\mathrm{m} 1$ in well-recovered patients with subcortical stroke. Stroke. 2014 Mar:45(3):788-93.

24. Jindahra P, Petrie A, Plant GT. The time course of retrograde trans-synaptic degeneration following occipital lobe damage in humans. Brain. 2012;135(2):534-41.

25. Duering $M$, Righart $R$, Csanadi $E$, Jouvent $E$, Hervé $D$, Chabriat $H$, et al. Incident subcortical infarcts induce focal thinning in connected cortical regions. Neurology. 2012 Nov 13;79(20):2025-8.

26. Walker PM, Salem DB, Lalande A, Giroud M, Brunotte F. Time course of NAA T2 and ADCW in ischaemic stroke patients: $1 \mathrm{H}$ MRS imaging and diffusionweighted MRI. J Neurol Sci. 2004;220(1-2):23-8.

27. Huo Y, Li O, Zhang W, Zou N, Li R, Huang S, et al. Total small vessel disease burden predicts functional outcome in patients with acute ischemic stroke. Front Neurol. 2019:10:808.

28. Held V, Szabo K, Bäzner H, Hennerici MG. Chronic small vessel disease affects clinical outcome in patients with acute striatocapsular stroke. Cerebrovascular diseases. 2012;33(1):86-91.

29. Zerna C, Yu AY, Modi J, Patel SK, Coulter Jl, Smith EE, Coutts SB. Association of white matter hyperintensities with short-term outcomes in patients with minor cerebrovascular events. Stroke. 2018;49(4):919-23.

\section{Publisher's Note}

Springer Nature remains neutral with regard to jurisdictional claims in published maps and institutional affiliations. 